\title{
The Lefschetz fixed point theorem for some non-compact multi-valued maps
}

\section{by}

Gilles Fournier (Montréal) and Lech Górniewicz (Gdańsk)

Abstract. A multi-valued map $p: X \rightarrow Y$ is called an admissible map provided there exists two maps $p: Z \rightarrow X$ and $q: Z \rightarrow Y$ such that

(i) $p$ is a Vietoris map,

(ii) $q p^{-1}(x) \subset \varphi(x)$ for all $x \in X$

In this paper, we consider admissible multi-valued maps $\varphi: X \rightarrow X$ which are locally compact and for which there exists a compact subset $K$ of $X$ such that, for any open neighbourhood $V$ of $K$,

we have $X=\bigcup_{n=0}^{\infty} p^{-n}(V)$. The Lefschetz fixed point theorem is proved, for these maps, in the following classes of spaces:

(i) open subsets of admissible linear spaces (in particular, locally convex spaces),

(ii) all NES (compact) spaces.

This result is applied to eventually compact maps and to asymptotically compact maps.

In this paper, all spaces are Hausdorff.

It is known [5], [6], that the Lefschetz fixed point theorem is true for compact admissible maps in the following classes of spaces:

(i) open subsets in admissible linear topological spaces (in the sense of Klee [9]) or, in particular, open subsets of locally convex topological spaces,

(ii) all NES(compact) spaces (in the sense of Hanner [8]).

In this note, we will be concerned with the extension of the above results for some non-compact admissible multi-valued maps.

In the single-valued case, these results were given by Fournier in [2], [3], [4].

In this paper, all spaces are Hausdorff.

1. Preliminaries. By a pair of spaces, $\left(X, X_{0}\right)$, we understand a pair consisting of a Hausdorff topological space $X$ and one of its subsets, $X_{0}$. A pair of the form $(X, \varnothing)$ will be identified with the space $X$. By a map, $f:\left(X, X_{0}\right) \rightarrow\left(Y, Y_{0}\right)$, we understand a continuous (single-valued) map $f: X \rightarrow Y$ satisfying the condition $f\left(X_{0}\right) \subset Y_{0}$.

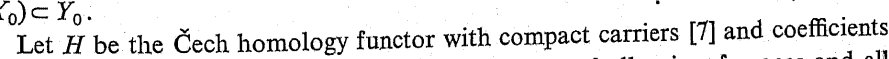
in the field of rational numbers $\mathscr{Q}$, from the category of all pairs of spaces and all maps between such pairs, to the category of graded vector spaces over $\mathscr{Q}$ and linear 
maps of degree zero. Thus $H\left(X, X_{0}\right)=\left\{H_{q}\left(X, X_{0}\right)\right\}$ is a graded vector space, $H_{q}\left(X, X_{0}\right)$ being the $q$-dimensional Čech homology with compact carriers of $X$ For a map $f:\left(X, X_{0}\right) \rightarrow\left(Y, Y_{0}\right), H(f)$ is the induced linear map $f_{*}=\left\{f_{* q}\right\}$, where $f_{* q}: H_{q}\left(X, X_{0}\right) \rightarrow H_{q}\left(Y, Y_{0}\right)$.

A non-empty space $X$ is called acyclic provided (i) $H_{q}(X)=0$ for all $q \geqslant 1$ and (ii) $H_{0}(X) \simeq$ 2. A map $p:\left(X, X_{0}\right) \rightarrow\left(Y, Y_{0}\right)$ is said to be a Vietoris map provided the following conditions are satisfied:

(i) $p$ is proper, i.e., for any compact $C$, the counter-image $p^{-1}(C)$ is also compact,

(ii) $p^{-1}\left(Y_{0}\right)=X_{0}$,

(iii) the set $p^{-1}(y)$ is acyclic for every $y \in Y$.

Note the following evident remark.

Remark 1.1. If $p:\left(X, X_{0}\right) \rightarrow\left(Y, Y_{0}\right)$ is a Vietoris map and $\left(B, B_{0}\right) \subset\left(Y, Y_{0}\right)$, then the map $\tilde{p}:\left(p^{-1}(B), p^{-1}\left(B_{0}\right)\right) \rightarrow\left(B, B_{0}\right)$ is also Vietoris, where $\tilde{p}(x)=p(x)$ for each $x \in p^{-1}(B)$.

THEOREM 1.2 (Vietoris-Begle Mapping Theorem [7]). If $p:\left(X, X_{0}\right) \rightarrow\left(Y, Y_{0}\right)$ is a Vietoris map, then the induced map $p^{*}: H\left(X, X_{0}\right) \sim H\left(Y, Y_{0}\right)$ is a linear isomorphism.

Let $u: E \rightarrow E$ be an endomorphism of an arbitrary vector space. Let us put $N(u)=\left\{x \in E \mid u^{n}(x)=0\right.$, for some $\left.n\right\}$, where $u^{n}$ is the $n$th iterate of $u$, and, $\tilde{E}$ $=E / N(u)$. Since $u(N(u)) \subset N(u)$, we have the induced endomorphism $\tilde{u}: \tilde{E} \rightarrow \tilde{E}$. We call $u$ admissible provided $\operatorname{dim} \tilde{E}<\infty$. Let $u=\left\{u_{q}\right\}: E \rightarrow E$ be an endomorphism of degree zero of a graded vector space $E=\left\{E_{q}\right\}$. We call $u$ a Leray endomorphism if (i) all $u_{q}$ are admissible and (ii) almost all $\tilde{E}_{q}$ are trivial. For such $u$, we define the (generalized) Lefschetz number $\Lambda(u)$ by putting

$$
\Lambda(u)=\sum_{q}(-1) \operatorname{tr}\left(\tilde{u}_{q}\right) .
$$

The following important property of the Leray endomorphisms is a consequence of the well known formula $\operatorname{tr}(u \circ v)=\operatorname{tr}(v \circ u)$ for trace.

Proposirion 1.3. Assume that, in the category of graded vector spaces, the following diagram commutes

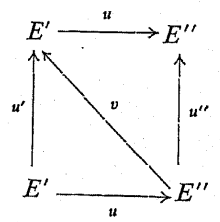

then, if $u^{\prime}$ or $u^{\prime \prime}$ is a Leray endomorphism, so is the other; and, in that case, $\Lambda\left(u^{\prime}\right)$ $=\Lambda\left(u^{\prime \prime}\right)$.
An endomorphism $u: E \rightarrow E$ of a graded vector space $E$ is called weakly-nilpotent if for every $q \geqslant 0$ and for every $x \in E_{q}$, there exists an integer $n$ such that $u_{q}^{n}(x)=0$. Since, for a weakly-nilpotent endomorphism $u: E \rightarrow E$, we have $N(u$ ) $=E$, so

Proposition 1.4. If $u: E \rightarrow E$ is a weakly-nilpotent endomorphism, then $\Lambda(u)=0$.

Let $f:\left(X, X_{0}\right) \rightarrow\left(X, X_{0}\right)$ be a map. If $f_{*}: H\left(X, X_{0}\right) \rightarrow H\left(X, X_{0}\right)$ is a Leray endomorphism, then $f$ is called a Lefschetz map. For such $f$, we define the Lefschetz number $\Lambda(f)$ of $f$ by putting $\Lambda(f)=\Lambda\left(f_{*}\right)$. Clearly, if $f$ and $g$ are homotopic, $f \sim g$, then $f$ is a Lefschetz map if and only if $g$ is a Lefschetz map; and, in this case, $\Lambda(f)=\Lambda(g)$.

Consider two maps $p, q:\left(Y, Y_{0}\right) \rightarrow\left(X, X_{0}\right)$, where $p$ is a Vietoris map. Let $p^{\prime}, q^{\prime}: Y \rightarrow X$ and $p^{\prime \prime}, q^{\prime \prime}: Y_{0} \rightarrow X_{0}$ be maps given by the same formula as $p$ and $q$ respectively. Observe that $p^{\prime}$ and $p^{\prime \prime}$ are Vietoris maps. Then, we have:

LEMMA 1.5. If two of those endomorphisms, $q_{*} p_{*}^{-1}, q_{*}^{\prime} p_{*}^{\prime-1}, q_{*}^{\prime \prime} p_{*}^{\prime \prime-1}$, are Leray endomorphisms, then so is the third; and, in that case,

$$
\Lambda\left(q_{*} p_{*}^{-1}\right)=\Lambda\left(q_{*}^{\prime} p_{*}^{\prime-1}\right)-\Lambda\left(q_{*}^{\prime \prime} p_{*}^{\prime \prime-1}\right) .
$$

Lemma 1.5 clearly follows from the exactness axiom for the Čech homology with compact carriers and coefficients in 2, and from (1.4) in [1].

2. Multi-valued maps. In the following, $\varphi, \psi$ will be reserved for multi-valued maps; the single-valued maps will be denoted by $f, g, p, q, \ldots$ Let $\varphi: X \rightarrow Y$ be a multi-valued map. We associate with $\varphi$ the following diagram of continuous maps:

$$
X \stackrel{p_{\varphi}}{\leftarrow} \Gamma_{\varphi} \stackrel{\dot{q}_{\varphi}}{\rightarrow} Y
$$

in which

$$
\Gamma_{\varphi}=\{(x, y) \in X \times Y \mid y \in \varphi(x)\}
$$

is the graph of $\varphi$ and the natural projections $p_{\varphi}$ and $q_{\varphi}$ are given by:

$$
p_{\varphi}(x, y)=x \text { and } q_{\varphi}(x, y)=y .
$$

The image of a subset $A \subset X$ under $\varphi$ is

$$
\varphi(A)=\bigcup_{x \in A} \varphi(x) .
$$

The counter-image of a subset $B \subset Y$ under $\varphi$ is

$$
\varphi^{-1}(B)=\{x \in X \mid \varphi(x) \subset B\} \text {. }
$$

A multi-valued map $\varphi: X \rightarrow Y$ is called upper semi-continuous (u.s.c.) provided (i) A multi-valued map $\varphi: X \rightarrow Y$ is called upper semi-coninu
$\varphi(x)$ is compact, for each $x \in X$, and (ii) for each open set $V \subset Y$, the counter-image $\varphi^{-1}(V)$ is an open subset of $X$.

Proposition 2.1 ([1]). If $\varphi: X \rightarrow Y$ is an u.s.c. map and $A$ is a compact subset of $X$, then the image $\varphi(A)$ of $A$ under $\varphi$ is compact. 
A multi-valued map $\varphi: X \rightarrow Y$ is called compact provided the image $\varphi(X)$ of $X$ under $\varphi$ is contained in some compact subset of $Y$. Let $\varphi: X \rightarrow Y$ be a multivalued map, $A$ a subset of $X$ and $B$ a subset of $Y$. If $\varphi(A) \subset B$, then the contraction of $\varphi$ to the pair $(A, B)$ is the multi-valued map $\varphi^{\prime}: A \rightarrow B$ defined by $\varphi^{\prime}(a)=\varphi(a)$ for each $a \in A$. A contraction of $\varphi$ to the pair $(A, Y)$ is simply the restriction $\left.\varphi\right|_{A}$ of $\varphi$ to $A$. Let $\varphi: X \rightarrow X$ be a multi-valued map and let $A$ be a subset of $X$. If $\varphi(A) \subset A$, then $A$ is called an invariant subset under $\varphi$. A point $x \in X$ is called a fixed point of $\varphi$ if $x \in \varphi(x)$.

Let $\varphi: X \rightarrow Y$ and $\psi: Y \rightarrow Z$ be two multi-valued maps. Then the composition of $\varphi$ and $\psi$ is a multi-valued map $\psi \circ \varphi: X \rightarrow Z$ defined by $\psi \circ \varphi(x)=\psi(\varphi(x))$ for each $x \in X$. For a multi-valued map $\varphi: X \rightarrow X$, we denote by $\varphi^{m}, m \geqslant 1$, the $m$ th iteration of $\varphi$ (i.e. $\varphi^{m}=\underbrace{\varphi \circ \ldots \circ \varphi}$ ); by $\varphi^{0}$, we denote the identity map $\operatorname{Id}_{X}$.

We note the following (see [7]):

Proposition 2.2. The composition of u.s.c. maps is also u.s.c.

From Proposition 2.2 and the definition of an u.s.c. map, we obtain:

LEMMA 2.3. Let $\varphi: X \rightarrow X$ be an u.s.c. map and let $U$ be an open invariant set under $\varphi$. Assume that, for every $x \in X$, there exists an integer $n$ such that $\varphi^{n}(x) \subset U$. Then, for every compact subset $K$ of $X$, there exists an integer $m$ such that $\varphi^{m}(K) \subset U$.

3. Admissible maps. An u.s.c. map $\varphi: X \rightarrow Z$ is said to be acyclic provided the set $\varphi(x)$ is acyclic for every point $x \in X$. We observe that if $\varphi: X \rightarrow Z$ is an acyclic map, then the natural projection $p_{\varphi}: \Gamma_{\varphi} \rightarrow X$ is a Vietoris map.

Let $\varphi: X \rightarrow Z$ be a multi-valued map; a pair $(p, q)$ (of single-valued, continuous) maps of the form $X \stackrel{p}{\leftarrow} Y \stackrel{q}{\leftarrow} Z$ is called a selected pair of $\varphi$ (written $(p, q) \subset \varphi$ ), if the following conditions are satisfied:

(i) $p$ is a Vietoris map,

(ii) $q\left(p^{-1}(x)\right) \subset \varphi(x)$ for each $x \in X$.

Remark 3.1. We observe that, if $\varphi$ is a compact map and $(p, q) \subset \varphi$, then $q$ is also compact.

DefinItIon 3.2 (see [5], [7]). An u.s.c. map $\varphi$ is called admissible provided there exists a selected pair $(p, q)$ of $\varphi$.

Every acyclic map and, in particular, every continuous single-valued map is admissible; for example, the pair $\left(p_{\varphi}, q_{\varphi}\right)$ is a selected pair of $\varphi$. We note that the composition of admissible maps is also admissible (see [5] or [7]).

Defintion 3.3. An admissible map $\varphi: X \rightarrow X$ is called a Lefschetz map provided, for each selected pair $(p, q) \subset \varphi$, the linear map $q_{*} p_{*}^{-1}: H(X) \rightarrow H(X)$
is a Leray endomorphism.

If $\varphi: X \rightarrow X$ is a Lefschetz map, then we define the Lefschetz set $\Lambda(\varphi)$ of $\varphi$ by putting

$$
\Lambda(\varphi)=\left\{\Lambda\left(q_{*} p_{*}^{-1}\right) \mid(p, q) \subset \varphi\right\}
$$

Remark 3.4 (see [5] or [7]). If $\varphi$ is an acyclic Lefschetz map, then the set $\Lambda(\varphi)$ is a singleton and, in this case, we shall denote it by $\Lambda(\varphi)$.

Let $\varphi: X \rightarrow X$ be an u.s.c. map and $U$ be an open invariant subset of $X$ under $\varphi$. Assume that, for every $x \in X$, there exists an integer $n$ such that $\varphi^{n}(x) \subset U$. Let $(p, q)$ be a selected pair of $\varphi$ of the form $X \stackrel{p}{\leftarrow} Y \stackrel{q}{\rightarrow} X$. Define $\tilde{p}:\left(Y, p^{-1}(U)\right)$ $\rightarrow(X, U), \tilde{q}:\left(Y, p^{-1}(U)\right) \rightarrow(X, U)$ by putting $\tilde{p}(y)=p(y)$ and $\tilde{q}(y)=q(y)$, for every $y \in Y$. Observe that $\tilde{p}$ is a Vietoris map. Then we have:

LEMMA 3.5. The endomorphism $\tilde{q}_{*} \tilde{p}_{*}^{-1}: H(X, U) \rightarrow H(X, U)$ is weakly-nilpotent.

Lemma 3.5 simply follows from Proposition 2.3 and the fact that $H$ is a homology functor with compact carriers, since $\left(q p^{-1}\right)^{n}(K) \subset \varphi^{n}(K)$, for each compact $K$ of $X$.

\section{Lefschetz multi-spaces.}

Definition 4.1. A Hausdorff space $X$ is called a Lefschetz multi-space for a class $\mathscr{2}$ of multi-valued maps, written $X \in L_{M}(\mathscr{Q})$, provided that any u.s.c. admissible map $\varphi: X \rightarrow X$ belonging to 2 , is a Lefschetz map and $\Lambda(\varphi) \neq\{0\}$ implies that $\varphi$ has a fixed point.

When 2 is the class of compact maps, we have the following theorem.

THEOREM 4.2. Are Lefschetz multi-spaces for the class of compact maps open subsets of

(i) admissible topological vector spaces (in the sense of Klee [9]); in particular, locally convex topological vector spaces,

(ii) NES (compact) (in the sense of Hanner [8]); in particular, ANR (metric).

Proof. It is evident from [5], Theorems 6.3 and 7.3, and the fact that an open subset of a NES (compact) is NES (compact).

Definition 4.3. A multi-valued map $\varphi: X \rightarrow X$ is said a compact absorbing contraction if there exists an open set $U$ of $X$ such that $\overline{\varphi(U)}$ is a compact of $U$ and $X \subset \bigcup_{i=0}^{\infty} \varphi^{-i}(U)$.

We state now our main theorem.

THEOREM 4.4. Let $X$ be a space such that every open set $V$ of $X$ satisfies $V \in L_{M}$ (compact), then $X \in L_{M}$ (compact absorbing contraction).

Proof. Let $\varphi: X \rightarrow X$ be an admissible map such that $\varphi \in 2$. Since $\varphi(U)$ $\subset \overline{\varphi(U)} \subset U$, consider $\varphi^{\prime}: U \rightarrow U$ the contraction of $\varphi$ to the pair $(U, U)$. Let $p, q$ : $Y \rightarrow X$ be a selected pair of $\varphi$ and, since $q\left(p^{-1}(U)\right) \subset \varphi(U) \subset U$, consider $p^{\prime}, q^{\prime}$ : $p^{-1}(U) \rightarrow U$ the contractions of $p$ and $q$ respectively. By Remark 1.1, $p^{\prime}$ is a Vietoris map; hence $\left(p^{\prime}, q^{\prime}\right)$ is a selected pair of $\varphi^{\prime}$, so $\varphi^{\prime}$ is admissible. Moreover $\varphi^{\prime}$ is compact, since $\overline{\varphi(U)}$ is a compact of $U$; so $\varphi^{\prime}$ is a Lefschetz map since $U \in L_{M}$ (compact). Consider the maps $p^{\prime \prime}, q^{\prime \prime}:\left(Y, p^{-1}(U)\right) \rightarrow(X, U) ; p^{\prime \prime}$ is a Vietoris map and, by Proposition 2.3, if $K$ is a compact subset of $X$, then there exists $n \in N$ such that $\varphi^{n}(K) \subset U$; so the map $q_{*}^{\prime \prime} p_{*}^{\prime \prime-1}$ is weakly-nilpotent, hence, by Proposition 1.4, 
$\Lambda\left(q_{*}^{\prime \prime} p_{*}^{\prime \prime-1}\right)=0$. By Lemma $1.5, q_{*}^{\prime} p_{*}^{\prime-1}$ is a Leray endomorphism and $\Lambda\left(q_{*} p_{*}^{-1}\right)$ $=\Lambda\left(q_{*}^{\prime} p_{*}^{\prime-1}\right)$. Hence $\varphi$ is a Lefschetz map and $\boldsymbol{\Lambda}(\varphi) \subset \boldsymbol{\Lambda}\left(\varphi^{\prime}\right)$. Now $\boldsymbol{\Lambda}(\varphi) \neq\{0\}$ implies that $\Lambda\left(\varphi^{\prime}\right) \neq\{0\}$; and, since $U \in L_{M}$ (compact), there exists $x \in X$ such that $x \in \varphi^{\prime}(x)=\varphi(x)$.

5. Compact attraction maps. Now, we define some classes of maps for which we will prove the Lefschetz theorem.

Definition 5.1. An u.s.c. multi-valued map $\varphi: X \rightarrow Y$ is called locally compact provided that, for each $x \in X$, there exists an open subset $V$ of $X$ such that $x \in V$ and the restriction, $\left.\varphi\right|_{Y}$, is compact.

Defintion 5.2. A multi-valued locally compact map $\varphi: X \rightarrow X$ is called eventually compact if there exists an iterate $\varphi^{n}: X \rightarrow X$ of $\varphi$ such that $\varphi^{n}$ is compact.

Defintrion 5.3. A multi-valued locally compact map $\varphi: X \rightarrow X$ is called compact attraction if there exists a compact $K$ of $X$ such that, for each open neighbourhood $V$ of $K$, we have $X \subset \bigcup_{i=0}^{\infty} \varphi^{-i}(V)$. The compact $K$ is then called an attractor for $\varphi$.

Defintion 5.4. A multi-valued locally compact map $\varphi: X \rightarrow X$ is called asymptotically compact if the set $C_{\varphi}=\bigcap_{n}^{\infty} \varphi^{n}(X)$ is a non-empty, relatively compact subset of $X$. The set $C_{\varphi}$ is called the center of $\varphi$.

Note that any multi-valued eventually compact map is a compact attraction and asymptotically compact map.

LEMMA 5.5. Any eventually compact map is a compact absorbing contraction map.

Proof. Let $\varphi: X \rightarrow X$ be an eventually compact map such that $K^{\prime}=\overline{\varphi^{n}(X)}$ is compact. Define $K=\bigcup_{i=0}^{n-1} \varphi^{i}\left(K^{\prime}\right)$, we have

$$
\varphi(K) \subset \bigcup_{i=1}^{n} \varphi\left(K^{\prime}\right) \subset K \cup \varphi^{n}(X) \subset K \cup K^{\prime} \subset K .
$$

Since $\varphi$ is locally compact, there exists an open neighbourhood $V_{0}$ of $K$ such that $L=\overline{\varphi\left(V_{0}\right)}$ is compact.

There exists a sequence $\left\{V_{1}, \ldots, V_{n}\right\}$ of open subsets of $X$ such that $L \cap \overline{\varphi\left(V_{i}\right)} \subset V_{i-1}$ and $K \cup \varphi^{n-i}(L) \subset V_{i}$ for all $i=1, \ldots, n$. In fact, if $K \cup \varphi^{n-i}(L)$ $\subset V_{\imath}$ and $0 \leqslant i<n$, since $K \cup \varphi^{n-i}(L)$ and $C V_{i} \cap L$ are disjoint compact sets of $X$, there exists an open subset $W$ of $X$ such that

$$
K \cup \varphi^{n-i}(L) \subset W \subset \bar{W} \subset V_{i} \cup C L .
$$

Define $V_{i+1}=\varphi^{-1}(W)$; since $\varphi(K) \cup \varphi\left(\varphi^{n-(i+1)}(L)\right) \subset K \cup \varphi^{n-i}(L) \subset W$, we have $K \cup \varphi^{n-(i+1)}(L) \subset V_{i+1}$; and $\varphi\left(V_{i+1}\right) \subset \bar{W} \subset V_{i} \cup C L$ implies $L \cap \overline{\varphi\left(V_{i+1}\right)} \subset V_{i}$. Beginning with $K \cup \varphi^{n}(L) \subset K \subset V_{0}$, we define, by induction $V_{1}, \ldots, V_{n}$ with the desired properties.
Putting $U=V_{0} \cap V_{1} \cap \ldots \cap V_{n}$, we have $K^{\prime} \subset K \subset U$ and

$$
\varphi(U) \subset \varphi\left(V_{0}\right) \cap \varphi\left(V_{1}\right) \cap \ldots \cap \varphi\left(V_{n}\right) \subset L \cap \overline{\varphi\left(V_{1}\right)} \cap \ldots \cap \overline{\varphi\left(V_{n}\right)},
$$

hence

$$
\left.\overline{\varphi(U)} \subset\left(L \cap \overline{\varphi\left(V_{1}\right)}\right) \cap \ldots \cap\left(L \cap \overline{\varphi\left(V_{n}\right.}\right)\right) \cap L \subset V_{0} \cap \ldots \cap V_{n-1} \cap V_{n}=U,
$$

but $\overline{\varphi(U)}$ is compact since $\overline{\varphi(U)} \subset L$. Moreover,

$$
X \subset \bigcup_{i=1}^{n} \varphi^{-i}\left(K^{\prime}\right) \subset \bigcup_{i=0}^{\infty} \varphi^{-i}(U) .
$$

Propositron 5.6. Any compact attraction map is a compact absorbing contraction map.

Proof. Let $\varphi: X \rightarrow X$ be a compact attraction map, $K$, a compact attractor for $\varphi$ and $W$, an open set of $X$ such that $K \subset W$ and $L=\overline{\varphi(W)}$ is compact. We have $L \subset X \subset \bigcup_{i=0}^{\infty} \varphi^{-i}(W)$ hence, since $L$ is compact, there exists $n \in N$ such that $L \subset \bigcup_{i=0}^{n} \varphi^{-i}(W)$. Define $V=\bigcup_{i=0}^{n} \varphi^{-i}(W)$; then

$$
X \subset \bigcup_{i=0}^{\infty} \varphi^{-i}(W) \subset \bigcup_{i=0}^{\infty} \varphi^{-i}(V),
$$

$$
\varphi(V) \subset \bigcup_{i=0}^{n} \varphi^{-i+1}(W) \subset \varphi(W) \cup V \subset L \cup V \subset V
$$

and

$$
\varphi^{n+1}(V) \subset \bigcup_{i=0}^{n} \varphi^{n-i+1}(W)=\bigcup_{j=0}^{n} \varphi^{j+1}(W) \subset \bigcup_{j=0}^{n} \varphi^{j}(L)
$$

which is compact and included in $V$, since $L \subset V$ and $\varphi(V) \subset V$ implies that $\varphi^{i}(L) \subset V$ for all $j \in N$. Consider the contraction $\varphi: V \rightarrow V$ of $\varphi ; \varphi^{\prime}: v \rightarrow U$ is an eventually compact map, since $V$ is an open set. By Lemma 5.5 , there exists an open set $U$ of $V$, hence of $X$, such that $\overline{\varphi^{\prime}(U)}=\overline{\varphi(U)}$ is a compact of $U$ and $V$ $\subset \bigcup_{n=0}^{\infty} \varphi^{\prime-n}(U) \subset \bigcup_{n=0}^{\infty} \varphi^{-n}(U)$; hence

$$
X \subset \bigcup_{i=0}^{\infty} \varphi^{-i}(W) \subset \bigcup_{i=0}^{\infty} \varphi^{-i}(V) \subset \bigcup_{n=0}^{\infty} \varphi^{-n}(U)
$$

From Theorem 4.4 and Proposition 5.6, we obtain:

CoRollary 5.7. Let $X$ be a space, if $V$ open in $X$ implies that $V \in L_{M}{ }^{\circ}$ (compact), then $X \in L_{M}$ (compact attraction) 
6. Asymptotically compact maps.

LEMMa 6.1. Let $\varphi: X \rightarrow X$ be an u.s.c. multi-valued map, $C_{\varphi}=\bigcap_{i=0}^{\infty} \varphi^{i}(X)$ and $V$ an open subset of $X$ such that $C_{\varphi} \subset V$. Then, for each compact $K$ of $X$, there exists $n \in N$ such that $\bigcap_{i=0}^{\infty} \varphi^{i}(K) \subset V$.

Proof. The family $\left\{\bigcap_{i=0}^{n} \varphi^{i}(K) \cap C V\right\}_{n \in N}$ of closed subsets of the compact $K$, has empty intersection, hence there exists a finite empty intersection.

LEMMA 6.2. Let $\varphi: X \rightarrow X$ be an u.s.c. multi-valued map, $C_{\varphi}=\bigcap_{i=0}^{\infty} \varphi^{i}(X), U_{\varphi}$ $=\left\{x \in X \mid \bigcup_{i=0}^{\infty} \varphi^{i}\right)$ is compact $\}$ and $V$, an open subset of $X$ such that $C_{\varphi p} \subset V$. Then $U_{\varphi} \subset \bigcup_{i=0}^{\infty} \varphi^{-i}(V)$.

Proof. Let $x \in U_{\varphi}, K=\bigcup_{n=0}^{\infty} \varphi^{n}(x)$ is compact; by Lemma 6.1 , there exists $n \in N$ such that $\varphi^{n}(x) \subset \bigcap_{i=0}^{\infty} \varphi^{i}(K) \subset V$.

Definition 6.3. A multi-valued map $\varphi: X \rightarrow X$ is called with compact orbits if $\bigcup_{n=0}^{\infty} \varphi^{n}(x)$ is relatively compact for every $x \in X$.

Proposition 6.4. Any asymptotically compact map with compact orbits, is a compact attraction map.

Proof. Let $\varphi: X \rightarrow X$ be an asymptotically compact map with compact orbits, then $U_{\varphi}=X$ so $\bar{C}_{\varphi}$ is a compact attractor for $\varphi$ and $\varphi$ is a compact attraction map.

Corollary 6.5. Let $X$ be a space, if $V$ open in $X$ implies that $V \in L_{M}$ (compact), then $X \in L_{M}$ (asymptotically compact with compact orbits).

LEMMA 6.6. Let $X$ be a space and $\varphi: X \rightarrow X$ an asymptotically compact map of center $C_{\varphi}$. Then, there exists an open subset $V$ of $X$ such that $C_{\varphi} \subset V, \varphi(V) \subset V$ and $\overline{\varphi(V)}$ is compact.

Proof. Let $U$ be an open set of $X$ such that $\bar{C}_{\varphi} \subset U$ and $K=\overline{\varphi(U)}$ is compact. By Lemma 6.1, there exists $n \in N$ such that $\bigcap_{i=0}^{n} \varphi^{i}(K) \subset U$. Define $V=\bigcap_{i=0}^{n} \varphi^{-i}(U)$. Since $\varphi\left(C_{\varphi}\right) \subset C_{\varphi}$, we have that $C_{\varphi} \subset V$. Moreover,

$$
\varphi(V) \subset \bigcap_{i=0}^{n} \varphi^{-i}(\varphi(U)) \subset \bigcap_{i=0}^{n} \varphi^{-i}(K)=\bigcap_{i=0}^{n} \varphi^{i-n}(K) \subset \varphi^{i-n}\left(\bigcap_{i=0}^{n} \varphi(K)\right) \subset \varphi^{i-n}(U),
$$

hence $\varphi(V) \subset \bigcap_{i=0}^{n-1} \varphi^{-i}(U) \cap \varphi^{-n}(U)=V$. Since $\varphi(V) \subset \varphi(U) \subset K, \overline{\varphi(V)}$ is com-

pact.
LEMMA 6.7. Let $X$ be a space and $\varphi: X \rightarrow X$ an asymptotically compact map of center $C_{\varphi}$, then $U_{\varphi}$ is open and $\varphi\left(U_{\varphi}\right) \cup C_{\varphi} \subset U_{\varphi}$.

Proof. By Lemma 6.6, let $V$ be an open set such that $C_{\varphi} \subset V, \varphi(V) \subset V$ and $K=\overline{\varphi(V)}$ is compact. Let us show that $U_{\varphi}=\bigcup_{i=0}^{\infty} \varphi^{-i}(V)$. By Lemma 6.2, we have that $U_{\varphi} \subset \bigcup_{i=0}^{\infty} \varphi^{-i}(V)$. Let $x \in \varphi^{-n}(V)$, then for all $m>n$,

$$
\varphi^{m}(x)=\varphi\left(\varphi^{k}\left(\varphi^{n}(x)\right)\right) \subset \varphi\left(\varphi^{k}(V)\right) \subset \varphi(V) \subset K,
$$

where $k=m-n-1$, so $\bigcup_{n=0}^{\infty} \varphi^{n}(x) \subset\left(\bigcup_{i=0}^{n} \varphi^{n}(x)\right) \cup K$, which is compact, hence $x \in U_{\varphi}$.

In these conditions, $\varphi^{\prime}: U_{\varphi} \rightarrow U_{\varphi}$, the contraction of $\varphi$ is defined and one is tempted to say that $\varphi^{\prime}$ is an asymptotically compact map with compact orbits, or that $\overline{C_{\varphi}}$ is a compact attractor of $\varphi^{\prime}$. Unfortunately neither of those hypothesis is true. The counterexample being complicated, is not presented here. However, one statement is true: $\varphi^{\prime}$ is a compact attraction map. Hence the following proposition.

Proposition 6.8. Let $\varphi: X \rightarrow X$ be an asymptotically compact map, then the contraction $\varphi^{\prime}: U_{\varphi} \rightarrow U_{\varphi}$ of $\varphi$ is a compact attraction map.

Proof. Since $\bar{C}_{\varphi}$ is a compact subset of $X, U_{\varphi}$ is an open set and $C_{\varphi} \subset U_{\varphi}$; by Lemma 6.1, there exists $n \in N$ such that $K=\bigcap_{i=0}^{n} \varphi \overline{\left(C_{\varphi}\right)} \subset U_{\varphi}$. Note that $K$ is compact and that $\varphi^{n}\left(C_{\varphi}\right) \subset \bigcap_{i=0}^{n} \varphi^{i}\left(C_{\varphi}\right) \subset K$, since $\varphi\left(C_{\varphi}\right) \subset C_{\varphi}$. Let $W$ be an open such that $K \subset W$, since $\varphi^{n}\left(C_{\varphi}\right) \subset W$, so $C_{\varphi} \subset \varphi^{-n}(W)=V$; by Lemma $6.2, U_{\varphi} \subset \bigcup_{i=0}^{\infty} \varphi^{-i}(V)$ hence $U_{\varphi} \subset \bigcup_{i=0}^{\infty} \varphi^{\prime-i}(W)$, since $\varphi\left(U_{\varphi}\right) \subset U_{\varphi}$. So $K$ is a compact attractor for $\varphi^{\prime}$, and $\varphi^{\prime}$ is a compact attraction map.

Corollary 6.9. Let $X$ be a space such that $V$ open in $X$ implies that $V \in L_{M}$ (compact), then if $\varphi: X \rightarrow X$ is an admissible asymptotically compact map, the contraction $\varphi^{\prime}: U_{\varphi} \rightarrow U_{\varphi}$ is a Lefschetz map; and $\Lambda\left(\varphi^{\prime}\right) \neq\{0\}$ implies that $\varphi$ has a fixed point.

\section{References}

[1] C. Bowszyc, Fixed point theorems for the pairs of spaces, Bull. Acad. Polon. Sci. 16 (1968), pp. $845-850$.

[2] G. Fournier, Théorème de Lefschetz, I-Applications éventuellement compactes, Bull. Acad. Polon. Sci. 6 (1975), pp. 693-701.

[3] - Théorème de Lefschetz, II - Applications d'attraction compacte, ibid., pp. 701-706.

6 - Fundamenta Mathematicae XCIV 
[4] G. Fournier, Théorème de Lefschetz, III - Applications asymptotiquement compactes, ibid. p. 707-713.

[5] - and L. Górniewicz, The Lefschetz fixed point theorem for multi-valued maps of nonmetrizable spaces, Fund. Math. 92 (1976), pp. 213-222.

[6] - and A. Granas, The Lefschetz Fixed Point Theorem for some classes of non-metrizable spaces, J. Math. Pures et Appliquées 52 (1973), pp. 271-284.

[7] L. Górniewicz, Homological methods in fixed point theory of multivalued maps, Dissertationes Math. 129 (1976), Warszawa.

[8] O. Hanner, Retraction and extension of mappings of metric and non-metric spaces, Ark. Math. 2 (1952), pp. 315-360.

[9] V. Klee, Leray-Schauder theory without local convexity, Math. Ann. 141 (1960), pp. 286-296. 